\title{
Prevalence of Rectal Carriage of Enterobacteriaceae Resistant to Beta-Lactam in Children Hospital Befelatanana in Madagascar 2016
}

\author{
Herinjaka Andriamandimbisoa ${ }^{\text {* }}$, Arinomenjanahary Rakotoarisoa ${ }^{1}$, \\ Tsiry Rasamiravak ${ }^{2}$, Andry Andrianarivelo ${ }^{1}$, Eric Farfour ${ }^{3}$, Mbola Rakotomahefa ${ }^{4}$ \\ and Andry Rasamindrakotroka ${ }^{2}$ \\ ${ }^{1}$ Laboratory of Bacteriology, CHU-JRA, Antananarivo, Madagascar \\ ${ }^{2}$ Laboratory of Training and Research in Medical Biology, Faravohitra, Madagascar \\ ${ }^{3}$ Laboratory clinical biology Hospital Foch, France \\ ${ }^{4}$ Pediatric department, Pediatric Hospital Befelatanana, Antananarivo, Madagascar \\ *Corresponding author
}

\section{A B S T R A C T}

The prevalence of antimicrobial drug resistant bacteria is increasing

Keywords

Beta-lactam, Children,

Enterobacteriaceae, Resistance.

Article Info

Accepted:

31 June 2017

Available Online:

10 July 2017 worldwide and represents a major thread in public health. We conducted a prospective and descriptive study during three months at the pediatric hospital Befelatanana Antananarivo on 2016. The aims of this study were to determine the prevalence of rectal carriage of Enterobacteriaceae beta lactam resistant on the children hospitalized during this period. During our study, one hundred and one patients were enrolled. The median age was 30 months. Main admitting diagnoses were respiratory $(63.4 \%)$, digestive (16.8\%), neurologic (13.9\%) and kidney or cardiac (5.9\%) disorders. Rectal swab yield growth of Enterobacteriaceae in 43 (42.6\%) children of which: $18(41.86 \%)$ E. cloacae, 10 (23.25\%) E. coli, 8 (18.6\%) K. pneumoniae, 7 (16.3\%) P. mirabilis. Overall, $32.6 \%$ of all strains were 3CG-R and 14 $(13.8 \%)$ children are colonized by a 3CG-R strain.

\section{Introduction}

The prevalence of antimicrobial drug resistant bacteria is increasing worldwide and represents a major thread in public health (Karanika et al., 2016). Although being on an isolated island people of Madagascar are not saved from this thread. Indeed, several study report now the presence of Multidrug resistant bacteria in Malagasy community. Since three decades, use of $\beta$-lactam has been trivial in health care center leading rapidly to the spread of resistant bacteria. Since 2000, third generation cephalosporin (3GC) was progressively introduced in Malagasy hospital to jugulate this resistance and subsequently, the first ESBL-producing Enterobacteriaceae (ESBL-PE) was isolated in Madagascar in 2005 from community-acquired urinary tract infections (Randrianirina et al., 2007). Thereafter, ESBL-PE has been isolated in patient hospitalized in surgical and intensive 
care units (Randrianirina et al., 2010) and in a pediatric department of a large teaching hospital in Antananarivo (Andriatahina et al., 2010). In 2009, Pasteur Institute of Madagascar reports the prevalence of ESBL$\mathrm{PE}$ carriage in the community (nonhospitalized patient) of Antananarivo as one of the highest $(10.10 \%)$ worldwide (Herindrainy et al., 2011). Furthermore, in children, ESBL-PE fecal carriage has been estimated at $21.2 \%$ in 2008 (Andriatahina et al., 2010). Thus, it's crucial to constantly evaluate reservoir of MDR strains in the largest Malagasy community. In order to contribute in this purpose, the present study evaluates the prevalence of Rectal Carriage of Gram-Negative Bacilli resistant to betalactam, including 3GC, among children attending three pediatric public hospitals in capital town of Madagascar.

\section{Materials and Methods}

Consecutive hospitalized children attending public hospitals of Befelatanana in capital town of Madagascar were included during a three months period between $1^{\text {st }}$ February and $30^{\text {th }}$ April 2016. Informed oral and written consent was obtained from at least one parent of each child before enrollment. Information about recent antibiotic use and previous hospitalization were also collected through brief questionnaires. Rectal swabs were collected by rotating the swab twice in the rectum of each patient. Collected specimens were sent directly to the Laboratory of Training and Research in Medical Biology Faravohitra and immediately plated on to DCL agar (Difco ${ }^{\circledR}$ Laboratories Inc., Detroit, MI, USA) and incubated for $24-48 \mathrm{~h}$ at $37^{\circ} \mathrm{C}$ in aerobic atmosphere. Every colony type was tested for Gram staining and oxidase activity. Oxidase-negative Gram-negative bacilli were identified with the API 20E system (bioMérieux, Marcy 1'Etoile, France). Susceptibility to beta-lactam of interest including amoxicilline, cefoxitine, cefotaxime, ceftazidime were determined by disk diffusion (Oxoid Ltd., Basingstoke, Hampshire, England) according to the Antibiogram Committee of the French society of Microbiology guidelines 2015. Reference Escherichia coli ATCC 25922 and Pseudomonas aeruginosa strains ATCC 27853 have been used as quality control.

\section{Results and Discussion}

One hundred and one patients were enrolled during the study period. The median age was 30 months [1 month - 15 years] and 82 $(81.2 \%)$ children were under 5 years old. The sex ratio (male/female) was 1.59. Main admitting diagnoses were respiratory $(63.4 \%)$, digestive (16.8\%), neurologic (13.9\%) and kidney or cardiac $(5.9 \%)$ disorders.

Rectal swab yield growth of Enterobacteriaceae in $43(42.6 \%)$ children of which: 18 (41.86\%) E. cloacae, 10 (23.25\%) E. coli, 8 (18.6\%) K. pneumoniae, 7 (16.3\%) P. mirabilis. Of these, 25 (58.1\%) were treated by antibiotic at the time of the sampling: including more than $2 / 3$ of children in whom a strain of E. cloacae or E. coli was isolated. Antibiotic prescribed were amoxicillin (64\%), amoxicillin plus clavulanic acid (16\%), ceftriaxone $(12 \%)$, or others (8\%). Among species naturally susceptible to amoxicillin, 13 of $17(76.4 \%)$ were resistant to this antibiotic, including 8/10 E. coli and 5/7 P. mirabilis strains. Among those naturally susceptible to cefoxitine, $11 / 25(44 \%)$ were resistant to this antibiotic. Overall, $32.6 \%$ of all strains were resistant to at least one 3CG and 14/101 (13.8\%) children are colonized by a strain resistant to $3 \mathrm{CG}$.

Pediatric population in Madagascar presents a high prevalence of beta-lactam resistance in Enterobacteriaceae: 3GC regardless of the mechanism of resistance, but also amoxicillin 
and cefoxitin. Prevalence of resistance to 3GC has been well described worldwide. In Madagascar, ESBL-PE prevalence has been estimated at $10.1 \%$ in adult community settings (Herindrainy et al., 2011) in 2009 and $21.2 \%$ in hospitalized children in 2008 (Randrianirina et al., 2010), which is higher than in our more recent work (2016). This difference could be explained by a median age (30 months) lower in our work compared to that of Andriatahina et al., (53 month). However, Andriatahina et al., focused specifically on ESBL-PE and therefore, the authors used selective agar media which are more sensitive than non-selective media used in this study. In fact, our approach was distinct as we assessed prevalence of rectal carriage of Enterobacteriaceae resistant to other beta-lactam as amoxicillin.

Table.1 Distribution and antibiotic resistance of Enterobacteriaceae collected and antibiotic treatment among children

\begin{tabular}{|c|c|c|c|c|c|}
\hline \multirow{2}{*}{ Enterobacteriaceae } & \multicolumn{4}{|c|}{ Antibiotic resistance to } & \multirow{2}{*}{$\begin{array}{l}\text { Antibiotic } \\
\text { treatment }\end{array}$} \\
\hline & AMX* & FOX* & CAZ* & CTX* & \\
\hline E. cloacae $(n=18)$ & $\begin{array}{l}\text { Natural } \\
\text { resistance }\end{array}$ & $\begin{array}{l}\text { Natural } \\
\text { resistance }\end{array}$ & $6(33.3 \%)$ & $5(27.7 \%)$ & $12(66.6 \%)$ \\
\hline E. coli $(n=10)$ & $8(80.0 \%)$ & $3(30.0 \%)$ & $2(20.0 \%)$ & $2(20.0 \%)$ & $7(70 \%)$ \\
\hline $\begin{array}{l}\text { K. pneumoniae } \\
(\mathbf{n = 8})\end{array}$ & $\begin{array}{l}\text { Natural } \\
\text { resistance }\end{array}$ & $3(37.5 \%)$ & $3(37.5 \%)$ & $3(37.5 \%)$ & $2(25 \%)$ \\
\hline P. mirabilis $(\mathrm{n}=7)$ & $5(71.4 \%)$ & $5(71.4 \%)$ & $2(28.6 \%)$ & $4(57.1 \%)$ & $3(42.8 \%)$ \\
\hline Total $(n=43)$ & $37(86.0 \%)$ & $23(53.5 \%)$ & $13(30.2 \%)$ & $14(32.6 \%)$ & $25(58.1 \%)$ \\
\hline
\end{tabular}

* AMX $=$ amoxicillin ; FOX : cefoxitin, CAZ : ceftazidime ; CTX : cefotaxime.

Furthermore, E. cloacae complex is the more frequent species isolated in our study whereas E. coli and K. pneumoniae are usually the more frequent species resistant to $3 \mathrm{GC}$. This singularity could be explained by the high proportion of children treated by antibiotic (Table 1) at the time of the sampling (more than $50 \%$ ). Prevalence of resistance to $3 \mathrm{GC}$ is particularly high in P. mirabilis, however, a small number of strains were included and we cannot rule out a punctual outbreak. We did not determine the mechanism of resistance to 3GC. 3GC resistance is generally mediated by ESBL in E. coli, K. pneumoniae and P. mirabilis and cephalosporinase in E. cloacae. Nevertheless, high prevalence of cefoxitin resistance may suggest implication of various mechanisms, either associated or alone, including: cephaslosporinase, impermeability, active efflux.
It has been demonstrated that a high prevalence of Enterobacteriaceae resistant to B-lactam in a pediatric population in Madagascar, suggesting an early acquisition of these strains. This work should be supplemented by specific research of ESBL$\mathrm{PE}$ as well as the analysis of the emerging carbapenemase producing Enterobacteriacea e.

\section{References}

Andriatahina $\mathrm{T}$, Randrianirina $\mathrm{F}$, Hariniana ER, Talarmin A, Raobijaona H, Buisson Y, et sal.2010. High prevalence of fecal carriage of extended-spectrum betalactamase-producing Escherichia coli and Klebsiella pneumoniae in a pediatric unit in Madagascar. BMC Infect Dis.10:204.

Herindrainy P, Randrianirina F, Ratovoson R, Ratsima Hariniana E, Buisson Y, Genel 
$\mathrm{N}$, et al.,2011. Rectal carriage of extended-spectrum beta-lactamaseproducing gram-negative bacilli in community settings in Madagascar. PLoS One.6 (7):e22738.

Karanika S, Karantanos T, Arvanitis M, Grigoras C, Mylonakis E.2016. Fecal Colonization with Extended-spectrum Beta-lactamase Producing Entero bacteriaceae and Risk Factors among Healthy Individuals: A Systematic Review and Metaanalysis. Clin Infect Dis.; 63(3):310-8.

Randrianirina F, Soares JL, Carod JF, Ratsima E, Thonnier V, Combe P et al.,
2007. Antimicrobial resistance among uropathogens that cause communityacquired urinary tract infections in Antananarivo, Madagascar. Antimicrob Chemother.59 (2):309-12. Randrianirina F, Vaillant L, Ramarokoto CE, Rakotoarijaona A, Andriamanarivo ML, Razafimahandry HC, et al.,2010. Antimicrobial resistance in pathogens causing nosocomial infections in surgery and intensive care units of two hospitals in Antananarivo, Madagascar. J Infect Dev Ctries.4 (2):74-82.

\section{How to cite this article:}

Herinjaka Andriamandimbisoa, Arinomenjanahary Rakotoarisoa, Tsiry Rasamiravak, Andry Andrianarivelo, Eric Farfour, Mbola Rakotomahefa and Andry Rasamindrakotroka. 2017. Prevalence of Rectal Carriage of Enterobacteriaceae Resistant to Beta-Lactam in Children Hospital Befelatanana in Madagascar 2016. Int.J.Curr.Microbiol.App.Sci. 6(7): 4532-4535. doi: https://doi.org/10.20546/ijcmas.2017.607.472 